\title{
Heat and SDS insensitive NDK dimers are largely stabilised by hydrophobic interaction to form functional hexamer in Mycobacterium smegmatis
}

\author{
Muthu Arumugam\# and Parthasarathi Ajitkumar $\bowtie$ \\ Department of Microbiology and Cell Biology, Indian Institute of Science, Bangalore -560012, Karnataka, India.
}

The primary structure and function of nucleoside diphosphate kinase (NDK), a substrate non-specific enzyme involved in the maintenance of nucleotide pools is also implicated to play pivotal roles in many other cellular processes. NDK is conserved from bacteria to human and forms a homotetramer or hexamer to exhibit its biological activity. However, the nature of the functional oligomeric form of the enzyme differs among different organisms. The functional form of NDKs from many bacterial systems, including that of the human pathogen, Mycobacterium tuberculosis (MtuNDK), is a hexamer, although some bacterial NDKs are tetrameric in nature. The present study addresses the oligomeric property of MsmNDK and how a dimer, the basic subunit of a functional hexamer, is stabilized by hydrogen bonds and hydrophobic interactions. Homology modeling was generated using the three-dimensional structure of MtuNDK as a template; the residues interacting at the monomermonomer interface of MsmNDK were mapped. Using recombinant enzymes of wild type, catalytically inactive mutant, and monomer-monomer interactive mutants of MsmNDK, the stability of the dimer was verified under heat, SDS, low $\mathrm{pH}$, and methanol. The predicted residues (Gln17, Ser24 and Glu27) were engaged in dimer formation, however the mutated proteins retained the ATPase and GTPase activity even after introducing single (MsmNDK- Q17A, MsmNDK-E27A, and MsmNDK-E27Q) and double (MsmNDK-E27A/Q17A) mutation. However, the monomer-monomer interaction could be abolished using methanol, indicating the stabilization of the monomer-monomer interaction by hydrophobic interaction.

Key words: nucleoside diphosphate kinase, Mycobacterium smegmatis, oligomerisation, dimer, hexamer, homology modeling, hydrophobic interaction, ANS fluorescence assay

Received: 24 January, 2013; revised: 14 May, 2013; accepted:

03 June, 2013; available on-line: 17 June, 2013

\section{INTRODUCTION}

Nucleoside diphosphate kinase (NDK) is a substrate non-specific enzyme that is primarily involved in the maintenance of nucleotide pools in all living systems, from bacteria to humans (Schaaper \& Mathews, 2013). NDK has also been implicated to play multiple roles in cellular metabolism, growth, and differentiation in a variety of organisms, including mycobacteria (Shankar et al., 1997; Biggs et al., 1990; Yoon et al., 2005). The primary, secondary, and tertiary structures of NDKs of diverse bacterial genera reveal high level of homology (Janin et al., 2000). However, the nature of the functional oligo- meric form (quaternary structure) of the enzyme differs among different bacterial genera. The functional entity of NDKs is hexamer in almost all lower and higher eukaryotes and among the bacterial systems, in Bacillus subtilis (Sedmak et al., 1971), Mycobacterium tuberculosis (Chen et al., 2002), Bacillus halodenitrificans (Chen et al., 2003), Halobacterium salinarum (Besir et al., 2005), and Pyrobaculum aerophilum (Pedelacq et al., 2005). However, Escherichia coli (Moynie et al., 2007), Salmonella typhimurium (Ingraham et al., 1978), Myxococcus xanthus (Williams et al., 1993), Streptomyces coelicolor (Brodbeck et al., 1996), Halomonas sp 593, and Pseudomonas aeruginosa (Tokunaga et al., 2008) have tetrameric NDK. The saprophytic mycobacterium, Mycobacterium smegmatis, although used as a model system to study mycobacterial biology differs from its pathogenic member, $M$. tuberculosis, in many conspicuous ways. In this regard, there is no information whether the functional form of NDK of M. smegmatis (MsmNDK) is also hexamer, and how the functional hexamer is structurally stabilized. In view of these facts and that NDK is an important enzyme, participating in a variety of cellular processes, the present study was undertaken to find out the functional oligomeric nature of MsmNDK.

The present finding elucidates the residues involved in the formation of dimers and the oligomeric status of MsmNDK by adopting different approaches, including: (i) homology modeling of MsmNDK dimer on the solved crystal structure of MtuNDK to find out the monomermonomer interacting residues (Chen et al., 2002); (ii) mutagenesis of these residues to generate single and double mutants to find out whether the disruption of these residues would affect dimerisation and thereby formation of higher order structure; (iii) chemical cross-linking of monomers using glutaraldehyde; (iv) exposure of the protein to heat and SDS, to disrupt subunit interactions; (v) influence of heat and methanol to find out hydrophobic interactions; and (vi) in silico analysis to map hydrophobic interactions, if any. The wild type MsmNDK and the mutant MsmNDK- H117Q, which lacks NTPase, NTP synthesising, and autophosphorylation activities (Arumugam \& Ajitkumar, 2012), were used as the controls in the experiments. The oligomeric profiles of the wild type protein and its structural mutants, prior to

e-mail: ajit@mcbl.iisc.ernet.in

\#Present Address: Scientist, Biotechnology division, National Institute for Interdisciplinary Science and Technology (NIIST), CSIR, Industrial Estate (PO), Trivandrum-695019, Kerala, India

Abbreviations: NDK, nucleoside diphosphate kinase; CCP4, collaborative computational project, 1994; ANS, 1-anilinonaphthalene8-sulfonic acid; CD, circular dichroism; PVDF, polyvinylidene fluoride; ECL, enhanced chemiluminescent and Hepes, N-2-Hydorxyethylpiperazine-N'-2-ethansulfonic acid. 
and subsequent to exposure to heat, SDS, low $\mathrm{pH}$, and methanol were verified using SDS/PAGE and crosslinking experiments. The observations showed that the MsmNDK monomers are held together as dimer mainly by hydrophobic interactions and due to the fact that the functional form of MsmNDK is hexamer.

\section{MATERIALS AND METHODS}

Bacterial strains, plasmids, and media. Plasmid pBS(KS) (Stratagene) and pQE30 (Qiagen) were used, respectively, for cloning and overexpression of Msmndk and its mutants. Escherichia coli JM109 and E. coli M15 cells, respectively, were used for the propagation of the wild type and mutant clones of Msmndk and for their overexpression. Luria broth with $100 \mu \mathrm{g}$ of ampicillin per $\mathrm{ml}$ was used for the selection and culturing of the recombinant clones.

Homology modeling of the MsmNDK dimer and prediction of contact residues. The amino acid sequence of MsmNDK was submitted to SWISS-MODEL (Arnold et al., 2006; Bordoli et al., 2009), server, generated modeled structure of MsmNDK monomer. The modeled monomer was then superimposed individually over six subunits of MtuNDK hexameric structure at $2.6 \AA$ resolution, using the programme ALIGN (Cohen, 1997), to obtain a hexameric MsmNDK model. The resulting model was validated through Ramachandran plot (Ramachandran et al., 1963). The distance between the monomers was calculated using the programme CONTACT of CCP4 (www.ccp4.ac.uk; Collaborative Computational Project, 1994). All the structural study figures were made using the Pymol molecular viewer software (www.pymol.org).

Generation of Msmndk mutants, overexpression, purification, and biochemical assays. Overlap extension PCR was used to generate three structural single mutants, namely MsmNDKQ17A, MsmNDK-E27A, and MsmNDK-E27Q, and the double mutant, MsmNDK-E27A/Q17A. Primers used for PCR are listed in Table S1 (all Suplementary Materials, designed by $\mathrm{S}$, at http://www.actabp. $\mathrm{pl} / \mathrm{pdf} / 2$ 2013/199-suppl.pdf). A 60 bp amplicon was generated using ORF specific forward (MsmNDKf2) and mutation-specific reverse primer (MsmNDKQ17Ar). Similarly, ORF specific reverse primer (MsmNDK-r2) and mutation-specific forward primer (MsmNDK-Q17Af) combination was used to yield a 360 bp product. These two mega primers were used for the generation of MsmNDK-Q17A mutant. Similarly, a 90 bp amplicon each was generated using ORFspecific forward (MsmNDK-f2) primer independently with the mutation-specific reverse primers, MsmNDK-E27Ar and MsmNDK-E27Qr. A 340 bp amplicon each was generated using ORF-specific reverse (MsmNDK-r2) primer independently with the mutation-specific forward primers, MsmNDK-E27Af and MsmNDK-E27Qf. These two sets of mega primers were used for the generation of MsmNDK-E27A and MsmNDK-E27Q mutants, using overlap extension PCR (Arumugam, 2010). The mutants were cloned in $\mathrm{pBS}-\mathrm{KS}$ and sequence verified. Subsequently, the MsmNDK-E27A mutant was used as a template to generate the double mutant, MsmNDK-E27A/Q17A, with the mutagenic primers, MsmNDK-Q17Af and MsmNDK-Q17Ar, and the ORF-specific primers, MsmNDK-f2 and MsmNDK-r2, in overlap extension PCR. The mutation was confirmed using DNA se- quencing. The three single structural mutants ( $\mathrm{MsmN}$ DK-Q17A, MsmNDK-E27A, and MsmNDK-E27Q) and a double (MsmNDK-E27A/Q17A) structural mutant were subcloned into pQE30 bacterial expression vector under lac promoter, which provides $6 \mathrm{xH}$ is tag at the $\mathrm{N}$-terminus of the fusion protein. The respective individual clones and the transformants are listed in Table 1. Cloning of the Msmndk gene and generation of the catalytically inactive mutant, MsmNDK-H117Q, have been described earlier (Arumugam \& Ajitkumar, 2012). The MsmNDK wild type and mutant proteins were overexpressed and purified, as previously described (Arumugam \& Ajitkumar, 2012). The ATPase and GTPase assays for the MsmNDK wild type and mutant proteins were carried out, as described earlier (Arumugam \& Ajitkumar, 2012).

The ${ }^{32} \mathrm{Pi}$ released from $\left[\gamma^{32} \mathrm{P}\right]-\mathrm{ATP}$ was quantified as counts per minute (CPM) for three independent experiments and the average values were plotted in form of a bar graph. The GTPase activity was determined by quantified as counts per minute (CPM) the $\left[\alpha^{3}{ }^{32} \mathrm{P}\right]-\mathrm{GDP}$ formed in three independent experiments and plotted as bar graph.

Circular dichroism (CD) spectroscopy. CD experiments were carried out using spectropolarimeter (JASCO J-715) measuring CD values over 200-260 nm wavelength range at every $0.5 \mathrm{~nm}$ interval, in $2 \mathrm{~mm}$ path-length cuvette. About $8.93 \mu \mathrm{M}$ protein was used in $1 \mathrm{ml}$ of $1 \mathrm{mM}$ Tris $/ \mathrm{HCl}$ ( $\mathrm{pH}$ 8.0) buffer containing 5 $\mathrm{mM} \mathrm{KCl}$, per assay at room temperature. The $\mathrm{CD}$ values are processed and plotted as graphs using Microsoft Excel 2003. CD spectrum was expressed in terms of mean residual ellipticity (Greenfield, 2006).

Chemical cross-linking of MsmNDK and its structural mutants. The cross-linking reaction was carried out in $20 \mu \mathrm{l}$ volume of $1 \times$ PBS buffer $(137 \mathrm{mM}$ $\mathrm{NaCl}, 2.7 \mathrm{mM} \mathrm{KCl}, 10 \mathrm{mM}$ sodium phosphate dibasic, $2 \mathrm{mM}$ potassium phosphate monobasic and $\mathrm{pH}$ of 7.4 , containing $2 \mu \mathrm{g}$ of $\mathrm{MsmNDK}$ wild type or the mutant proteins at $4^{\circ} \mathrm{C}$ or $37^{\circ} \mathrm{C}$. The cross-linking reaction was initiated with the addition of varying concentrations of glutaraldehyde $(0 \%, 0.001 \%, 0.005 \%, 0.01 \%, 0.02 \%$ and $0.1 \%$ ). The reaction was allowed to proceed for $20 \mathrm{~min}$ and then quenched with the addition of $0.2 \mathrm{M}$ Tris/ $\mathrm{HCl}, \mathrm{pH} 7.4$, for $15 \mathrm{~min}$ at room temperature. The individual samples were resolved in $12 \%$ SDS/PAGE, along with protein size markers, and the gel was silver stained.

Exposure of MsmNDK and its mutants to low $\mathrm{pH}$. In order to verify the dimer stability for MsmNDK or its mutant proteins, the protein samples were subjected to two different acidic $\mathrm{pH}$. Six micrograms of MsmNDK wild type, MsmNDK-H117Q, MsmNDK-Q17A, MsmNDK-E27Q, MsmNDK-E27A or MsmNDK Q17A/E27A, were incubated in sodium acetate $(\mathrm{pH} 4.0)$ or HEPES-KOH ( $\mathrm{pH}$ 6.5) buffer for 2 $\mathrm{h}$ on ice. The proteins were then resolved in $12 \%$ SDSPAGE along with protein size markers and stained with coomassie brilliant blue.

Prediction of hydrophobic patches in MsmNDK (hydropathy plot). Hydropathy plot was constructed using the Kyte-Doolittle hydropathy analysis programme (Kyte \& Doolittle, 1982) at the ExPASy molecular biology server (http://www.expasy.ch/cgibin/protscale.pl). The hydropathy plot was repeated using another software, WinPep protein sequence analyser, as well (Hennig, 1999). The MsmNDK amino acid sequence was fed into the above-mentioned programme with the window size 9, based on which it 
Table 1. Bacterial Strains, Plasmids and NDK structural mutant Constructs

\begin{tabular}{lll}
\hline Reagents & Genotype & Reference/Source \\
\hline
\end{tabular}

\section{Bacterial strains}

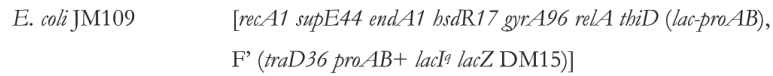

Yanish-Perron et al., 1985

QIAGEN

$\begin{array}{lll}\text { Plasmids } & & \\ \text { pBS(KS) } & \text { Cloning vector, Amp } & \text { Messing } \text { et al., } 1981 \\ \text { pQE30 } & \text { Expression vector with N-terminal 6xHis-tag } & \text { QIAGEN } \\ \text { Constructs } & & \text { Arumugam \& Ajitkumar, 2012 } \\ \text { pBS(KS)-MsNDK } & \text { MsNDK ORF cloned between KpnI and EcoRI } & \text { Arumugam \& Ajitkumar, 2012 } \\ \text { pQE30-MsNDK } & \text { MsNDK ORF cloned between KpnI and PstI } & \text { Arumugam \& Ajitkumar, 2012 } \\ \text { pBS(KS)-MsNDK-H117Q } & \text { MsNDK-H117Q cloned between KpnI and EcoRI } & \text { Arumugam \& Ajitkumar, 2012 } \\ \text { pQE30-MsNDK-H117Q } & \text { MsNDK-H117Q cloned between KpnI and PstI } & \text { This Study } \\ \text { pBS(KS)-MsNDK-Q17A } & \text { MsNDK-Q17A cloned between KpnI and EcoRI } & \text { This Study } \\ \text { pQE30-MsNDK-Q17A } & \text { MsNDK Q17A cloned between KpnI and PstI } & \text { This Study } \\ \text { pBS(KS)-MsNDK-E27A } & \text { MsNDK-E27A cloned between KpnI and EcoRI } & \text { This Study } \\ \text { pQE30-MsNDK-E27A } & \text { MsNDK E27A cloned between KpnI and PstI } & \text { This Study } \\ \text { pBS(KS)-MsNDK-E27Q } & \text { MsNDK-E27Q cloned between KpnI and EcoRI } & \text { This Study } \\ \text { pQE30-MsNDK-E27Q } & \text { MsNDK E27Q cloned between KpnI and PstI } & \text { This Study } \\ \text { pBS(KS)-MsNDK-Q17A/E27Q } & \text { MsNDK-Q17A/E27Q cloned between KpnI and EcoRI } \\ \text { pQE30-MsNDK-Q17A/E27Q } & \text { MsNDK MsNDK-Q17A/E27Q cloned between KpnI and PstI } & \text { This Study }\end{array}$

computed the hydrophobic score for individual amino acids and plotted as graph.

ANS fluorescence assay. The 1-anilinonaphthalene8-sulfonic acid (ANS) fluorescence assay was carried out as previously described (Semisotnov et al., 1991). In
A

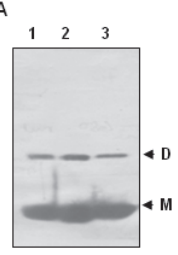

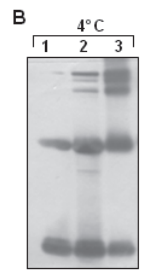
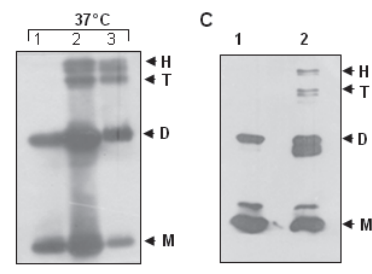

Figure 1. (A) Western blot for MsmNDK and its catalytic mutant MsmNDK-H117Q.

Three $\mu \mathrm{g}$ of MsmNDK wild type (Lane 1), three $\mu \mathrm{g}$ (Lane 3) or six $\mu \mathrm{g}$ (Lane 2) of MsmNDK-H117Q mutant protein.

(B) Oligomerisation of MsmNDK in the presence of chemical cross linker.

Cross-linking experiment was carried out using $2 \mu \mathrm{g}$ of purified recombinant MsmNDK in $1 \mathrm{X}$ PBS at $37^{\circ} \mathrm{C}$ or $4^{\circ} \mathrm{C}$. Lane, 1 . MsmNDK in presence of $0.001 \%$ Glutaraldehyde, Lane, 2. MsmNDK in presence of $0.01 \%$ Glutaraldehyde and Lane, 3. MsmNDK in presence of $0.1 \%$ Glutaraldehyde.

(C) Western blot profile of MtuNDK with (Lane, 2) or without $0.01 \%$ Glutaraldehyde (Lane, 2 ).

$\mathrm{M}$, Monomer; D, Dimer; $\mathrm{T}$, Tetramer and $\mathrm{H}$, Hexamer. In the presence of the amino group specific cross-linker glutaraldehyde, MsmNDK could form, in addition to dimer, higher order oligomers like tetramer and hexamer at $4^{\circ} \mathrm{C}$ as well as at $37^{\circ} \mathrm{C}$ as evident by immunoblot (Fig. 1B). brief, 1:50 ratio (1 $\mu \mathrm{M}$ NDK and $50 \mu \mathrm{M}$ ANS) of MsmNDK wild type and ANS was incubated in $200 \mu \mathrm{l}$ of freshly prepared $20 \mathrm{mM}$ Tris $/ \mathrm{HCl}(\mathrm{pH} 8)$ buffer. These were incubated separately or in the presence of increasing concentration of methanol $(0 \%, 20 \%, 40 \%, 60 \%$, $80 \%$ and $100 \%$ ) in the same cuvette for $5 \mathrm{~min}$ at $25^{\circ} \mathrm{C}$. The fluorescence values of buffer-blank were subtracted from the experimental CD values. Excitation wavelength of $360 \mathrm{~nm}$ was used, and emission spectra were measured from $400 \mathrm{~nm}$ to $600 \mathrm{~nm}$. Slit widths were kept at 3 $\mathrm{nm}$ for excitation and at $5 \mathrm{~nm}$ for emission acquisition. While plotting, fluorescence values were plotted on Yaxis with wavelength on the $\mathrm{X}$-axis in a wavelength-scan mode.

Methanol treatment and immunoblotting. Immunoblotting was performed with $3 \mu \mathrm{g}$ of the MsmNDK wild type protein. The protein was incubated with $0 \%, 20 \%, 40 \%, 60 \%, 80 \%$ and $100 \%$ (v/v) methanol. Then, $1 \times$ SDS buffer was added, the methanol was allowed to air dry, the protein samples were boiled and loaded on 12\% SDS/PAGE. The PVDF membrane, onto which the proteins were electro-transferred, was blocked for $3 \mathrm{~h}$ with the $1 \times$ PBS buffer containing $0.1 \%$ Tween-20 and 5\% skimmed milk. The PVDF membrane was incubated with affinity-purified anti-MsmNDK polyclonal antibody (1:10000 dilutions) for $3 \mathrm{~h}$. The membrane was washed three times with wash buffer (1X PBS containing $0.1 \%$ Tween-20), incubated with anti-rabbit HRP secondary antibody (as per Sigma guidelines), and developed using ECL reagent (Sigma). 


\section{RESULTS}

\section{MtuNDK and MsmNDK are hexameric and, forms SDS stable dimer}

SDS/PAGE electrophoresis of recombinant, soluble, MsmNDK (wild type) and MsmNDK-H117Q mutant (hereinafter called H117Q) proteins showed that both the wild type (Fig. 1A, lane 1) and the mutant proteins (Fig. 1A, lanes 2 and 3 ) form homodimer. In the presence of the amino group specific cross-linker glutaraldehyde, MsmNDK could form, in addition to dimer, higher order oligomers like tetramer and hexamer at $4^{\circ} \mathrm{C}$ as well as at $37^{\circ} \mathrm{C}$ as evident by immunoblot (Fig. 1B). For comparison purpose, similar experiments were carried out for purified recombinant MtuNDK at $37^{\circ} \mathrm{C}$, and showed the formation of higher order oligomers (Fig. 1C \& S6). Whereas, the purified protein without chemical cross-linker showed only dimer population and not higher order oligomers in MsmNDK (Fig. 1A, lane 1) and MtuNDK (Fig. 1C, lane 1).

\section{Homology modeling and identification of monomer- monomer interacting residues in MsmNDK dimer interfacer}

In order to identify the residues that hold the MsmNDK dimer species, which is stable under heat and SDS, a model was constructed for the MsmNDK dimer subunit. The crystal structure of hexameric MtuNDK was known at $2.6 \AA$ resolution (Chen et al., 2002) and there exists $81 \%$ identity between the primary structures of MtuNDK and MsmNDK. Therefore, the MtmNDK structure $(1 \mathrm{~K} 44)$ was used as a template to model the MsmNDK structure. The MsmNDK amino acid sequence was submitted to the SWISS-MODEL server, to obtain the MsmNDK monomeric modeled structure (Fig. S1A). The resulting model was validated using the Ramachandran plot (Fig. S1B), which showed that the majority of the modelled amino acid residues have fallen in the permissible region of the Ramachandran plot. Therefore, the SWISS-MODEL of MsmNDK is reliable, and it can be used to identify the contact residues that hold two monomeric subunits together to form dimer, the basic subunit for the formation of a functional NDK hexamer. The modeled MsmNDK monomers were superimposed over the six subunits of the MtNDK hexameric structure using the Align software, as mentioned under the Materials and Methods section, to get the hexameric MsmNDK structure (Fig. S1C).

A possible arrangement of the dimer within the hexamer of MsmNDK was explored using visual inspection of hexamer and contact residues between monomers. The contact distance between two monomers was evaluated in terms of hydrogen bonds, using the Contact programme (http://www.ccp4.ac.uk). A distance ranging from $2.5 \AA$ to $3.6 \AA$ between the hydrogen donor and acceptor was considered for the hydrogen bond. Based on these criteria, possible interactions between residues were investigated within two chains that can form dimer. The possible interactions and the monomers that can form dimer within the hexamer are presented (Fig. 2A). The exact dimeric interface between two monomers has been mapped and the contact residues have been identified (Fig. 2B). Contact residues and their distances are presented in Table 2.

\section{Generation of MsmNDK structural mutants, overexpression and purification}

According to the MsmNDK dimer model, three residues Gln-17, Ser-24 and Glu-27 are found to have surface contact with other monomer in the dimer. Multiple sequence alignment was performed for the predicted residues (Gln17, Ser24 and Glu27) among mycobacterial or other diverse bacterial genera (Fig. S2). The following single mutants, Q17A, E27A and E27Q, and a double mutant Q17A/E27A, were generated using site-directed mutagenesis (Table 1), overexpressed, and purified (Fig. S3) as described by Arumugam and Ajitkumar, (2012).

\section{Biochemically active MsmNDK structural mutants}

Mutants of the interacting residues, MsmNDK-Q17A, MsmNDK-S24A, MsmNDK-E27Q, MsmNDK-E27A, and the double mutant, MsmNDK-Q17A/E27A, will Hereinafter be referred to as Q17A, S24A, E27Q, E27A, and Q17A/E27A, respectively. Far UV circular dichroism spectroscopic analyses of the purified recombinant
A

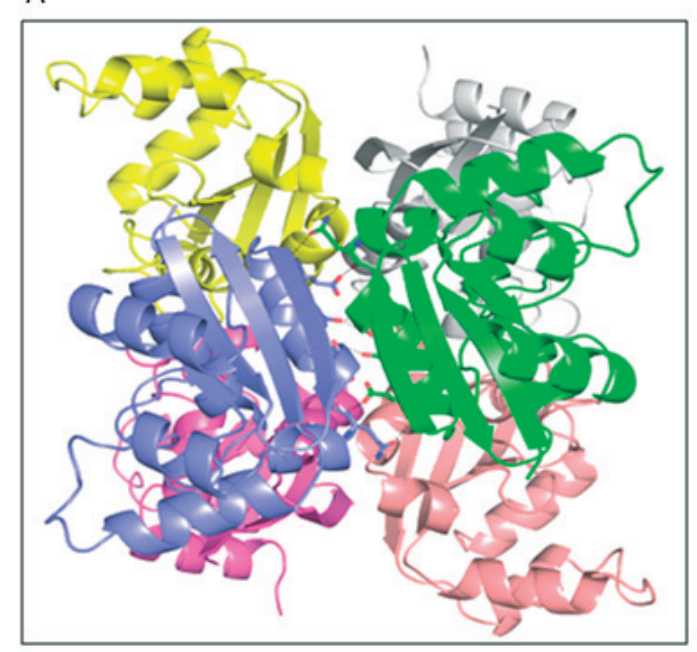

B

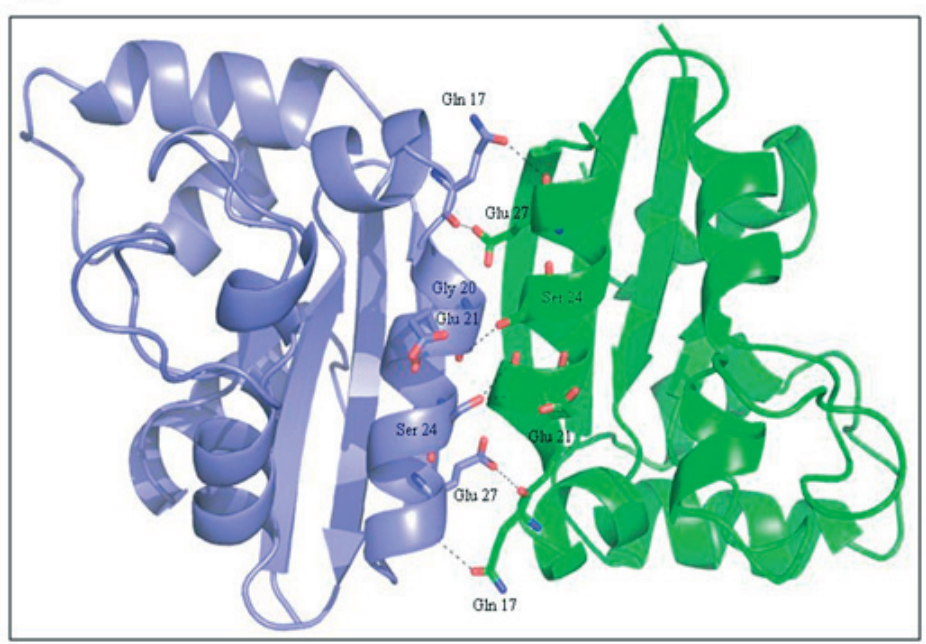

Figure 2. The predicted dimer interface contact residues between two monomeric subunits of MsmNDK. (A) Dimer arrangement within the hexameric form of MsmNDK. (B) Illustration of the predicted dimer interface contact residues between two monomeric subunit of MsmNDK. 
Table 2. Interaction Between two Monomeric subunits ( $R$ and $T$ ) and their contact Distance in $\AA$

\begin{tabular}{|c|c|c|c|c|}
\hline \multicolumn{2}{|l|}{ Monomeric Subunit R } & \multicolumn{2}{|l|}{ Monomeric Subunit T } & \multirow{2}{*}{ Distance (in Å) } \\
\hline Name of Amino acid & Position & Name of Amino acid & Position & \\
\hline Glutamine & 17 & Glutamic acid & 27 & 3.64 \\
\hline Glutamine & 17 & Glutamic acid & 27 & 3.36 \\
\hline Serine & 24 & Glutamic acid & 21 & 3.10 \\
\hline Serine & 24 & Glycine & 20 & 3.66 \\
\hline Glutamic acid & 27 & Glutamine & 17 & 3.70 \\
\hline
\end{tabular}

monomer- monomer interaction single mutant proteins, Q17A, S24A, E27Q, E27A, and the double mutant, Q17A/E27A, showed secondary structure alteration in the mutant proteins, with loss of helical content, as compared to the wild type MsmNDK protein (Arumugam, 2010). Nevertheless, all single mutants and the double mutant showed ATPase activity (Fig. S4A). Similarly, all single mutants, Q17A, S24A, E27Q, and E27A, showed GTPase activity, comparable to that of the wild type MsmNDK (Fig. S4B). However, the double mutant, Q17A/E27A, showed marginally reduced GTPase activity, with respect to its corresponding single mutants, Q17A or E27A. The double mutant, Q17A/E27A, showed marginally reduced $\left[\alpha-{ }^{32} \mathrm{P}\right]-\mathrm{GDP}$ formation. The very low GTPase activity was observed for the catalytically inactive mutant, H117Q.

\section{Wild type and structural mutants of MsmNDK are not altered at low $\mathrm{pH}$}

The SDS/PAGE analysis (followed by coomassie brilliant blue and silver stain) of single and double structural mutants, showed the presence of small percentage of dimer, which was stable even in the presence of the anionic detergent SDS and heat (boiling of the samples before loading onto SDS/PAGE). All single mutants,

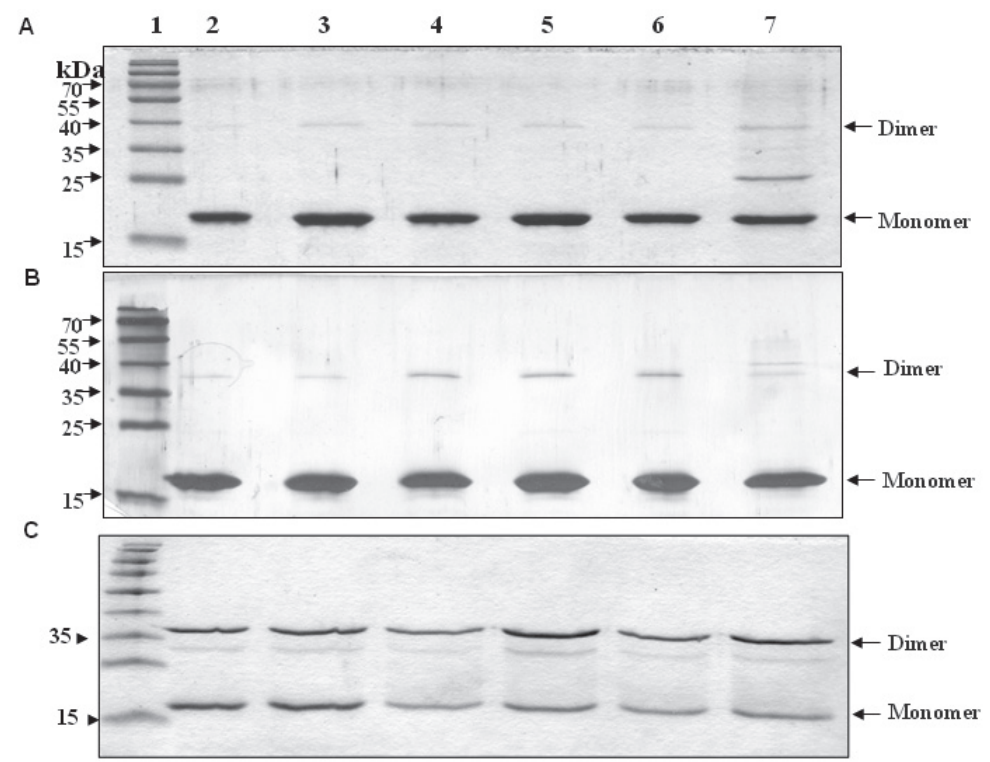

Figure 3. MsmNDK wild type and its mutant: SDS and heat stable dimer.

Coommassie stained (A) or silver stained (B) gel profile of purified recombinant MsmNDK and its mutant proteins. (C) Effect of $\mathrm{pH}$ (6.5) on the stability of MsmNDK wild type, catalytically inactive mutant and its structural mutants. The faint band lower to the dimer band is nonspecific Lane 1, Protein size marker; Lane 2, MsmNDK Wt; Lane 3, MsmNDK-H117Q; Lane 4, MsmNDK-Q17A; Lane 5, MsmNDK-E27Q; Lane 6, MsmNDK-E27A; Lane 7, Double mutant MsmNDK-E27A/Q17A. The heat, SDS, pH (6.5) stable dimer was indicated.
H117Q, Q17A, S24A, E27A, and $\mathrm{E} 27 \mathrm{Q}$, showed dimer population (Fig. 3A \& 3B lanes 3, $4,5,6)$. The double mutant, Q17A/E27A, also showed dimer population (Fig. 3A \& 3B, lane, 7). All the experiments on dimerisation of MsmNDK and its mutants were carried out at $\mathrm{pH} 8.0$, at which even the double mutant showed stable dimer population. However, dimer formation by MsmNDK single and double mutants, comparable to that of the wild type, could be observed at $\mathrm{pH} 6.5$ (Hepes-KOH buffer) (Fig. 3C) and even at $\mathrm{pH} 4.0$ (sodium acetate buffer) (Arumugam, 2010). It suggests that acidic pH does not alter the dimer forming capabilities of neither the wild type nor the single or double mutants of MsmNDK.

\section{Cross-linking shows the presence of natural dimers of MsmNDK and its mutants}

Crosslinking using increasing concentrations of glutaraldehyde, from $0.001 \%$ to $0.02 \%(0 \%, 0.001 \%, 0.005 \%$, $0.01 \%, 0.02 \%$ and $0.1 \%$ ), showed increase in the dimer population of both the wild type (Fig. S5A, lanes 2-5) and the catalytically inactive mutant MsmNDK-H117Q (Fig. S5B, lanes 2-5). Commensurate with the increase in the dimer species, a gradual decrease in the monomer population was observed at increasing glutaraldehyde concentration. The dimer formation drastically dropped at $0.1 \%$ glutaraldehyde concentration, and all of the NDK were converted into higher oligomeric forms, which did not get resolved properly in $12 \%$ SDS/PAGE, but were accumulated near the well (Fig. S5A, lane 6; Fig. S5B, lane 6). The single mutants, Q17A, E27Q, and $\mathrm{E} 27 \mathrm{~A}$, and the double mutant, Q17A/E27A, also behaved similarly to the wild type protein. Here, an increasing dimer population could also be observed (Fig. S5C-F), when the glutaraldehyde concentration was increased, but it dropped at the high concentration of $0.1 \%$ glutaraldehyde. The concomitant reduction of monomer was observed in all of the single mutants (Fig. S5C-F).

\section{Identification of hydrophobic interactions on the monomer- monomer interface}

The maximum hydrophobic score of 0.716 was observed for MsmNDK Val72, whereas the minimum score (0.264) was observed for the Ala51 position. Based on the hydrophobic scores from the hydrophobic plot (Fig. 4A), hydrophobic patches were mapped in the MsmNDK primary structure. The corresponding amino acids, hydrophobic scores were presented (Fig. 4B). The analysis of the modeled dimer revealed the role of hydrophobic interaction in holding the two mono- 


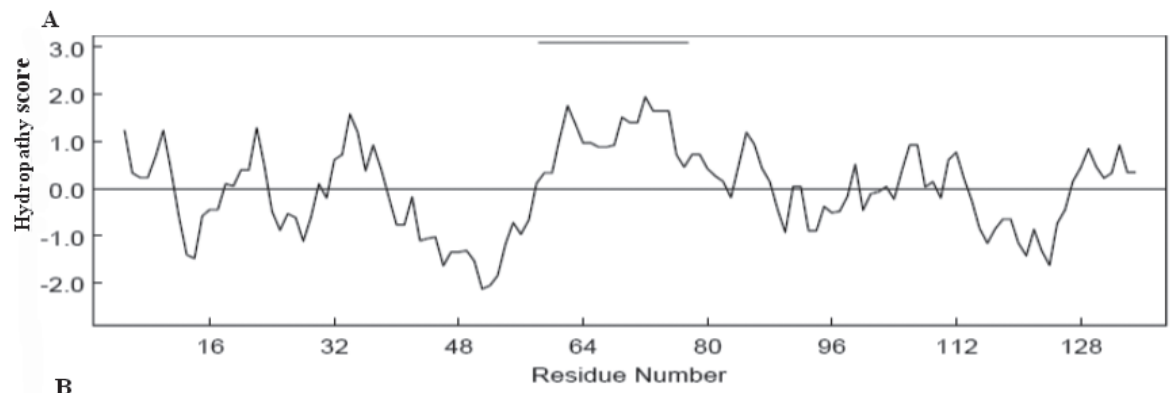

B

\begin{tabular}{|l|c|l|c|}
\hline Firs Hydrop athy patch & Hydrophobic Score & Second Hydrop athy patch & Hydrophobic Score \\
\hline Leucine (33) & 0.580 & Proline (70) & 0.656 \\
\hline Alanine (34) & 0.675 & Leucine (71) & 0.656 \\
\hline Alanine (35) & 0.632 & Valine (72) & 0.716 \\
\hline Leucine (36) & 0.542 & Alanine (73) & 0.683 \\
\hline Glutam ate (37) & 0.602 & Alanine (74) & 0.683 \\
\hline Leucine (38) & 0.546 & Isoleucine (75) & 0.683 \\
\hline Lysine (39) & 0.480 & Valine (76) & 0.580 \\
\hline Aspragine (40) & 0.415 & Glutam ate(77) & 0.551 \\
\hline & & Glycine (78) & 0.512 \\
\hline & & Proline (79) & 0.537 \\
\hline & & Arginine (80) & 0.547 \\
\hline
\end{tabular}

Figure 4. Hydropathy plot for MsmNDK.

(A) Hydropathy plot was computed for MsmNDK amino acid sequence using WinPep software (Version 3. 01). The amino acid residues (X-axis) and the Kyte and Doolittle, Hydropathy score (Y-axis) were plotted. (B) The hydrophobic patches residues and the hydophobic score. Numbers in the parenthesis indicates position of in MsmNDK primary structure.

mers together. The dimer interface consisted of residues from 33 to 40 and residues from 70 to 80 , forming two anti-parallel $\beta$-strands (Fig. 5A). The residues forming these $\beta$-strands were found to be hydrophobic residues (Fig. 4B). These two $\beta$-strands form a continuous $\beta$-sheet on the dimer interface. The hydrogen bonding interactions, which were already described, and the hydrophobic interactions occur at two levels or layers, which are placed one below the other on the dimer interface (Fig. 5B). Thus, the MsmNDK monomer-monomer interface seems to contain hydrophobic interactions (dotted spheres) (Fig. 5B), in addition to the hydrogen bonds (dash lines).

\section{Verification of hydrophobic interactions on the monomer-monomer interface}

The unique property of 1-anilinonaphthalene-8-sulfonic acid (ANS), which shows fluorescence with a quantum yield of more than 0.5 when adsorbed on hydrophobic regions in protein, as compared to nonfluorescence in water (quantum yield $\sim 0.002$ ) (Stryer, 1965), was used to examine the role of hydrophobic patches found in the MsmNDK monomer-monomer interface. The buffer containing $50 \mu \mathrm{M}$ ANS showed fluorescence peak, in contrast to the baseline of buffer alone (Fig. 6A). The buffer containing MsmNDK alone showed base line fluorescence, but the addition of ANS enhanced the fluorescence intensity of ANS to a considerable extent (Fig. 6A), indicative of ANS binding to hydrophobic patches of MsmNDK. The ANS fluorescence intensity was found to increase gradually from the base level $(0 \%$ methanol) to higher intensity at $100 \%$ methanol, indicating that the methanol increased the surface exposure of the hydrophobic patch of Ms$\mathrm{mNDK}$, thereby facilitating more binding by ANS, resulting in enhanced fluorescence (Fig. 6B). This experiment indicated that the hydrophobic patches play a major role in maintaining the dimeric form of the MsmNDK protein.

Immunoblotting was performed for the MsmNDK wild type protein incubated in the presence of increasing concentrations of methanol. The heat- and SDS-stable dimer disappeared from 40\%-methanol concentration onwards (Fig. 6C). It indicated that the $\mathrm{MsmN}$ DK dimer was maintained mostly by hydrophobic interaction, in addition to hydrogen bonds in solution, to perform its biological function.

\section{DISCUSSION}

This present study shows that NDK from $M$. smegmatis forms stable dimer, which is resistant to heat and anionic detergent SDS. Extensive analyses on the genetic, biochemical, and crystallographic aspects of NDKs from diverse organisms have shown that NDKs from bacterial systems remain tetramer, which is their functional form (Williams et al., 1993; Janin et al., 2000), while those
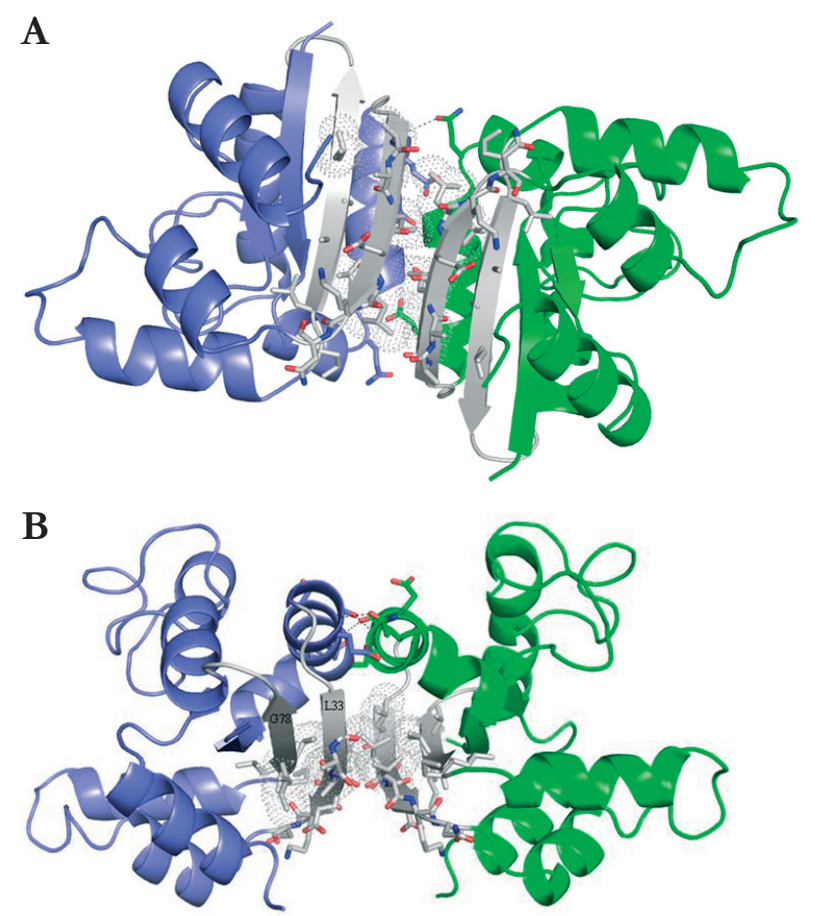

Figure 5A. The dimer interface of MsmNDK involving hydrogen bond (dash lines) as well as hydrophobic interaction (dotted spheres). The strand at the interface is indicated as gray colour. Figure 5B. The same dimer interface represented in another orientation, which shows the holding of dimer by hydrogen bond and hydrophobic interaction clearly. 
A

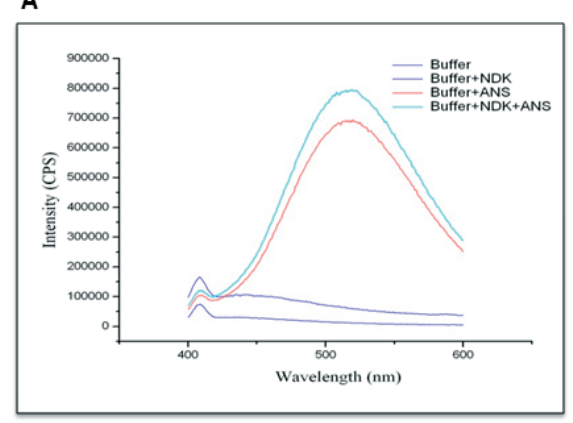

B
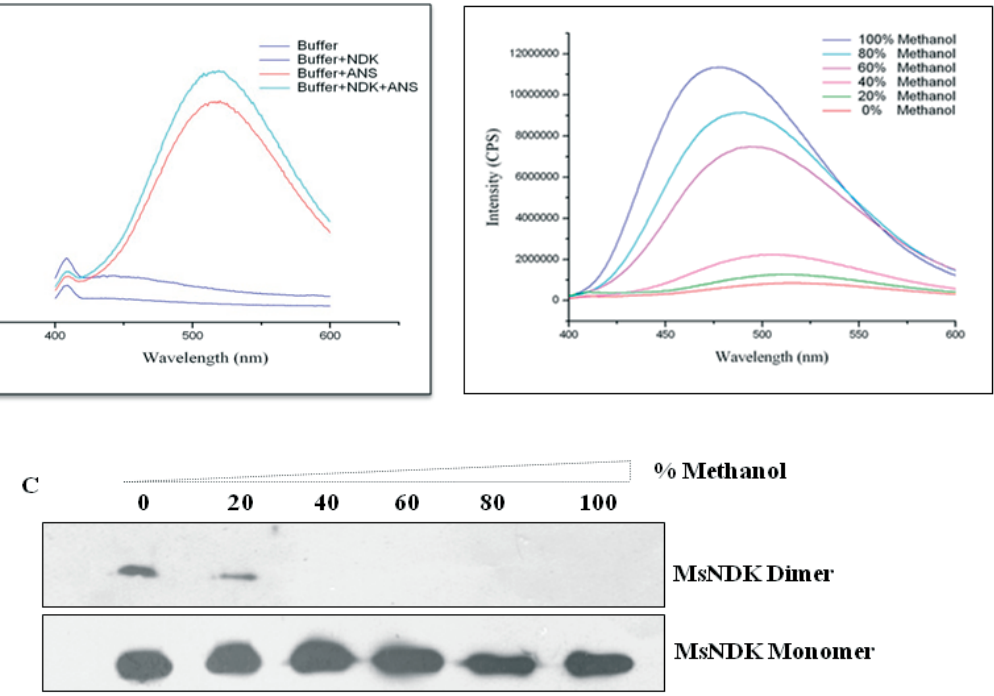

Figure 6. ANS binding assay for MsmNDK wild type protein in the presence of organic solvent methanol.

(A) Controls to show that ANS is binding to NDK and either buffer or NDK or Buffer containing MsmNDK is not fluorescing by itself. (B) MsmNDK in the presence of increasing concentration of methanol (see the inset legends in figure). (C) Western blot showing the presence or absence of MsmNDK dimer in the presence of increasing concentration of methanol.

from eukaryotes and $M$. tuberculosis are found in functional hexameric form (Chen et al., 2000; Janin et al., 2000). It was, shown for the first time that NDK from the moderate halophile, Halomonas sp. 593 (HaNDK) forms dimer different to that of $P$. aeruginosa NDK, which comes in a tetramer form (Yonezawa et al., 2001; Yonezawa et al., 2007). More recently, Bacillus antbracis NDK (BanNDK) crystal structure has been solved, and it reveals that BanNDK also exists in a hexamer form, like its eukaryotic and M. tuberculosis homologues. These hexamers consist of two SDS-stable trimers to form active hexameric form (Misra et al., 2009). Immunobloting, protein sequencing of MsmNDK dimer, and chemical cross-linking experiments, along with homology modelling, revealed that MsmNDK also exists in a hexamer form. Since the wild type MsmNDK and the structural mutants consisted of SDS- and heat-stable dimer, but not trimers, glutaraldehyde cross-linking experiments did not show trimer species, rather only dimer, tetramer, and hexamer species. The possibility of trimer formation and two such trimers forming a hexamer was not found tenable. Thus, probably three homodimers might be forming an active hexameric form.

According to the MsmNDK dimer model, three residues Gln17, Ser24, and Glu27 were found to have surface contact with other monomer, in the dimer. These interacting residues were found to be conserved across NDKs from different organisms. Multiple sequence alignment, using ClustalW (http://www. ebi.ac.uk/clustalw) and information from NCBI, a genome database, showed very good conservation for Glu27 among bacterial, Arabidopsis, Drosophila, and Human, or across diverse bacterial genera (Fig. S2). This indicates the importance of Glu 27 in the surface contact between monomers. Similarly, Gln17 and Ser24 are conserved well among mycobacterial species, again indicating the importance of these residues in the monomer-monomer contact.

In present study it was observed that $G \ln 17$, Ser24 and Glu27 at N-terminal of MsmNDK, interact with each other. In agreement with the above-presented findings, the Gly residue at the $\mathrm{N}$-terminus of NDK from Bacillus anthracis (G19), $M$. xanthus (G21) and $P$. aeruginosa (G21) is well conserved and that mutation of this Gly19 residue to Val19 leads to the disruption of the oligomeric association and a complete abolition of the enzyme activity has been reported (Williams et al., 1993; Misra et al., 2009). The Gly19 in BanNDK is in the vicinity of Glu20 and Ile18, and the Gly19 to Val19 mutation looks a a drastic one, as the rmsd reported in its work to show a big difference (Misra et al., 2009). The MsmNDK model predicts that the Ser24 residue on the dimer interface has contact with the conserved Gly20 (equivalent to the Gly19 of BanNDK) and the conserved Glu21 (equivalent to Glu20 of BaNDK) (Table 2). The efforts to obtain the Ser24 to Ala24 mutant were not successful, and therefore the effect of losing the contact with Gly20 cannot be mentioned here now. On the other hand, Halomonas sp. 593 NDK (HaNDK) forms a dimeric assembly, whereas Pseudomonas aeruginosa NDK (PaeNDK) forms a tetrameric assembly. The Glu134 to Ala mutation in HaNDK resulted in the conversion of the native dimeric structure to the tetramer assembly. Conversely, Ala134 to Glu change in PaeNDK led to the conversion from the tetramer to the dimer assembly (Tokunaga et al., 2008). This indicated that the single amino acid residue at position 134 is critical for the formation of the the native oligomeric structure of NDK. By modeling the structure of HaNDK and PaeNDK based on the crystal structure of Myxococcus NDK, it was shown that Glu134 exerts sufficient repulsive forces to disrupt the dimer-dimer interaction and prevent the formation of the tetramer. In MsmNDK, the residue at position 134 is Pro, which is conserved in NDKs of mycobacterial species.

In general, thermophilic proteins/enzymes have a higher content of hydrophobic amino acids than that in the proteins of mesophiles, and these residues can increase the rigidity and hydrophobicity of proteins (Chakravarty \& Varadarajan, 2000), thereby increasing their structural and functional integrity at high temperatures. Higher number of hydrophobic groups in thermophilic enzymes destabilises the unfolded forms gives more rigidity with increases in the temperature (Ikai et al., 1980; Britton et al., 1995). Reports from various research groups show that hydrophobic core and aliphatic side chains could also be responsible for protein stability at high temperatures (Argos et al., 1977; Lindsay, 1995; Haney et al., 1991; Vieille et al., 2001; Fütter et al., 2004; Irimia et al., 2004). In view of these studies, there is a 
possibility that the stability of the MsmNDK dimer in heat and SDS might be due to the hydrophobic core present in the wild type and in the mutants. This experiment indicated that dimer formation was not abolished even in the double mutant, where both residues, Gln17 and Glu27 of the wild type protein, were mutated to Ala. Thus, either single or double mutant of the residues identified could not abolish the homodimerisation of MsmNDK. MsmNDK structural mutants (three single and one double mutant) retained their ATPase and GTPase activities, which suggested that the mutations might not have affected oligomerisation capability of the protein. It indicated that the MsmNDK dimer might be stabilised by factors other than hydrogen/ionic bonds formed by the residues identified for mutagenesis.

The ANS binding assay in the presence of increasing concentrations of methanol, in combination with mapping of hydrophobic patches using hydropathy plot and western blotting for the dissociated monomers, confirmed the existence of hydrophobic patches in MsmNDK. Two $\beta$-strands, one of which that starts from amino acids $33-40$ and the other that spans across residues 70 to 80 , have hydrophobic residues, which exist topologically in parallel to the predicted hydrogen bonds. A similar study reports that bromelain:Fab' complex binds in 1:1 stoichiometry, and that the disassociation of this complex starts from $5 \%(\mathrm{v} / \mathrm{v})$ methanol, which is steady till $20 \%$, followed by significant dissociation, which becomes complete at $40 \%(\mathrm{v} / \mathrm{v})$ methanol concentration (Gupta et al., 2006). Thus, hydrophobic interactions play a prominent role in maintaining the dimeric forms of MsmNDK.

\section{CONCLUSIONS}

Mycobacterium Smegmatis NDK forms the functional hexamer through three homo dimers. Dimer interface of MsmNDK was mapped because it was considered to be a basic subunit to form the functional MsMNDK hexamer. Gln17, Ser24 and Glu27, which lie in the $\mathrm{N}$-terminal region of the protein, were found interact (Hydrogen bond) with each other to form a stable dimer. Using site directed mutagenesis, single and double structural mutants of the above-mentioned residue has been generated to verify the above findings. The ANS binding assay, in the presence of increasing concentrations of methanol, in combination with mapping of hydrophobic patches using the hydropathy plot and western blotting for the dissociated monomers, confirmed the existence hydrophobic patches in MsmNDK. Two $\beta$-strands, one of which that starts from amino acids 33-40 and the other that spans across residues 70 to 80 , have hydrophobic residues, which exist topologically in parallel to the predicted hydrogen bonds. The ANS fluorescence binding assay and organic solvent to disrupt the dimer confirms that hydrophobic interactions play a prominent role in maintaining the dimeric form of MsmNDK, which in turn is involved in the formation of a functional hexamer.

\section{Acknowledgements}

This work was supported in part by the Centre of Excellence in Tuberculosis Research part-grant from DBT. MA was a CSIR SRF. M. Selvaraj of Molecular Biophysics Unit, IISc are acknowledged for their help in instructing homology modeling and the prediction of interacting amino acid residues. The infrastructural facilities provided by the DBT Programme Support - Pathogen Biology - to Biological Sciences Division, IISc, UGC-CAS and DST-FIST support to the MCB Dep't., IISc, are also acknowledged.

\section{Competing interests}

The author(s) declare that they have no competing interests.

\section{REFERENCES}

Argos P, Rossman MG, Grau UM, Zuber H (1979) Thermal stability and protein structure. Biochemistry 18: 5698-5703.

Arnold K, Bordoli L, Kopp J, Schwede T (2006) The SWISS-MODEL Workspace: A web-based environment for protein structure homology modelling. Bioinformatics 22: 195-201.

Arumugam M (2010) Structural, functional and transcriptional analysis of Nucleoside Diphosphate Kinase of Mycobacterium smegmatis, doctoral thesis, Indian Institute of Science, Banglore, India. 41-69.

Arumugam M, Ajitkumar P (2012) Histidine 117 in the His-Gly-SerAsp motif is required for the biochemical activities of nucleoside diphosphate kinase of Mycobacterium smegmatis. Open Biochem J 6: 71-77.

Besir H, Zeth K, Bracher A, Heider U, Ishibashi M, Tokunaga M, Oesterhelt D (2005) Structure of a halophilic nucleoside diphosphate kinase from Halobacterium salinarum. FEBS Lett 579: 65956600 .

Biggs J, Hersperger E, Steeg PS, Liotta LA, Serán A (1990) A Drosophila gene that is homologous to a mammalian gene associated with tumor metastasis codes for a nucleoside diphosphate kinase. Cell 63: 933-940.

Bordoli L, Kiefer F, Arnold P, Benkert K, Battey J, J Schwede T (2009) Protein structure homology modeling using SWISS-MODEL workspace. Nature Protocols 4: 1-13.

Britton KL, Baker PJ, Borges KM, Engel PC, Pasquo A, Rice DW, Robb FT, Scandurra R, Satillman TJ, Yip KS (1995) Insights into thermalstability from a comparison of the glutamate dehydrogenases from Pyrococcus furiosus and Thermococcus litoralis. Eur I Biochem 229: 688-695.

Brodbeck M, Rohling A, Wohlleben W, Thompson CJ, Susstrunk U (1996) Nucleoside-diphosphate kinase from Streptomyces coelicolor. Eur J Biochem 239: 208-213.

Chakravarty S, Varadarajan R (2000) Elucidation of determinants of protein stability through genome sequence analysis. FEBS 470: 65-69.

Chen Y, Morera, S, Mocan M, Lascu I, Janin J (2002) X-ray structure of Mycobacterium tuberculosis nucleoside diphosphate kinase. Proteins 47: 556-557.

Chen CJ, Liu MY, Chang T, Chang WC, Wang BC, Gall JL (2003) Crystal structure of a nucleoside diphosphate kinase from Bacillus halodenitrificans: coexpression of its activity with a Mn-superoxide dismutase. I Struct Biol 142: 247-255.

Cohen G (1997) ALIGN: a program to superimpose protein coordinates, accounting for insertions and deletions. J Appl Crystallogr 30: 1160-1161.

Greenfield NJ (2006) Using circular dichroism collected as a function of temperature to determine the thermodynamics of protein unfolding and binding interactions. Nature Protocols 6: 2527-2533.

Gupta P, Saleemuddin M, Khan RH (2006) Hydrophobic interactions are the prevalent force in bromelain:Fab' complex. Biochemistry (Moscow) 71: S31-S37.

Haney PJ, Badger JH, Buldak GL, Reich CI, Woese CR, Olsen GJ (1999) Thermal adaptation analyzed by comparison of protein sequences from mesophilic and extremely thermophilic Methanococcus species. Biochem 96: 3578-3583.

Hennig L (1999) WinGene/WinPep: User-friendly software for the analysis of aminoacid sequences. BioTechniques 26: 1170-1172.

Ikai A (1980) Thermostability and aliphatic index of globular proteins. J Biochem (Tokyo) 88: 1895-1898.

Ingraham JL, Ginther CL (1978) Nucleoside diphosphokinase from Salmonella typhimurium. Methods Enzymol 51: 371-375.

Irimia A, Vellieux FM, Madern D, Zaccai G (2004) The 2.9A resolution crystal structure of malate dehydrogenase from Archaeoglobus fulgidus: mechanisms of oligomerisation and thermal stabilisation. $J$ Mol Biol 335: 343-356.

Janin J, Dumas C, Morera S, Xu Y, Meyer P, Chiadmi M, Cherfils J (2000) Three- dimensional structure of nucleoside diphosphate kinase. J Bioenerg Biomembr 32: 215-225.

Lindsay JA (1995) Is thermophily a transferable property in bacteria? Crit Rev Microbiol 21: 165-174.

Messing J, Crea R, Seeburg PH (1981) A system for shotgun DNA sequencing. Nucleic Acids Res 9: 309-321. 
Misra G, Aggarwal A, Dube D, Zaman MS, Singh Y, Ramachandran R (2009) Crystal structure of the Bacillus anthracis nucleoside diphosphate kinase and its characterization reveals an enzyme adapted to perform under stress conditions. Proteins 76: 496-506.

Moynie L, Giraud MF, Georgescauld F, Lascu I, Dautant A (2007) The structure of the Escherichia coli nucleoside diphosphate kinase reveals a new quaternary architecture for this enzyme family. Proteins 67: $755-765$.

Pedelacq JD, Waldo GS, Cabantous S, Liong EC, Terwilliger TC (2005) Structural and functional features of an NDP kinase from the hyperthermophile crenarchaeon Pyrobaculum aerophilum. Protein Science 14: 2562-2573.

Schaaper RM, Mathews CK (2013) Mutational consequences of dNTP pool imbalances in E. coli. DNA Repair 12: 73-79.

Sedmak J, Ramaley R (1971) Purification and properties of Bacillus subtilis nucleoside diphosphate kinase. J Biol Chem 246: 5365-5372.

Semisotnov GV, Rodionova NA, Razgulyaev OI, Uversky VN, Gripas AF, Gilmanshin RI (1991) Study of the "molten globule" intermediate state in protein folding by a hydrophobic fluorescent probe. Biopolymers 31: 19-128.

Shankar S, Hershberger CD, Chakrabarty AM, (1997) The nucleoside diphosphate kinase of Mycobacterium smegmatis: identification of proteins that modulate specificity of nucleoside triphosphate synthesis by the enzyme. Mol Microbiol 24: 477-487.

Snapper SB, Melton RE, Mustafa S, Kieser T, Jacobs Jr WR (1990) Isolation and characterization of efficient plasmid transformation mutants of Mycobacterium smegmatis. Mol Microbiol 4: 1911-1919.

Stryer L (1965) The interaction of a naphthalene dye with apomyoglobin and apohemoglobin: A fluorescent probe of non-polar binding sites. J Mol Biol 13: 482-495.
Tokunaga H, Ishibashi M, Arisaka F, Arai S, Kuroki R, Arakawa T, Tokunaga M (2008) Residue 134 determines the dimer-tetramer assembly of nucleoside diphosphate kinase from moderately halophilic bacteria. FEBS Lett 582: 1049-1054.

Tokunaga H, Ishibashi M, Arisaka F, Arai S, Kuroki R, Arakawa T, Tokunaga M (2008) Residue 134 determines the dimer-tetramer assembly of nucleoside diphosphate kinase from moderately halophilic bacteria. FEBS Lett 582: 1049-1054.

Vieille C, Epting KL, Kelly RM and Zeikus JG (2001) Bivalent cations and amino-acid composition contribute to the thermostability of Bacillus licheniformis xylose isomerase. Eur J Biochem 268: 6291-6301.

Williams RL, Oren DA, Munoz-Dorado J, Inouye S, Inouye M, Arnold E (1993) Crystal structure of Myxococcus xanthus nucleoside diphosphate kinase and its interaction with a nucleotide substrate at $2.0 \AA$ resolution. J Mol Biol 234: 1230-1247.

Yanisch-Perron C, Vieira J, Messing J (1985) Improved M13 phage cloning vectors and host strains: nucleotide sequences of the M13mp18 and pUC19 vectors. Gene 33: 103-119.

Yonezawa Y, Ichiizutsu K, Tokunaga H, Maeda H, Arakawa T, Tokunaga M (2007) Dimeric structure of nucleoside diphosphate kinase from moderately halophilic bacterium:contrast tothe tetrameric Pseudomonas counterpart. FEMS Microbiol Lett 268: 52-58.

Yonezawa Y, Y Tokunaga H, H Ishibashi H, Tokunaga M (2001) Characterisation of nucleoside diphosphate kinase from moderately halophilic eubacteria. Biosci Biotechnol Biochem 65: 2343-2346.

Yoon JH, Singh P, Lee DH, Qiu J, Cai Q, O’Connor TR, Chen Y, Shen B, Pfeifer GP (2005) Characterisation of the 3'-5' exonuclease activity found in human nucleoside diphosphate kinase 1 (NDK1) and several of its homologues. Biochemistry 44: 15774-15786. 